\title{
Effect of Soil Characteristics on Potato Tuber Minerals Composition of Selected Kenyan Varieties
}

\author{
Martha N. Wekesa ${ }^{1}$, Michael W. Okoth ${ }^{1}$, George O. Abong, ${ }^{1}$, Jane Muthoni ${ }^{2} \&$ Jackson N. Kabira $^{2}$ \\ ${ }^{1}$ Department of Food Science, Nutrition and Technology, University of Nairobi, Nairobi (Kangemi), Kenya \\ ${ }^{2}$ National Potato Research Centre (KARI), Tigoni, Limuru, Kenya \\ Correspondence: Martha Wekesa, Department of Food Science, Nutrition and Technology, University of Nairobi, \\ P.O. Box 29053-00625, Nairobi (Kangemi), Kenya. E-mail: martha.wekesa@yahoo.com
}

Received: July 23, 2014 Accepted: September 18, 2014 Online Published: November 15, 2014

doi:10.5539/jas.v6n12p163 URL: http://dx.doi.org/10.5539/jas.v6n12p163

\begin{abstract}
The current study was set up to evaluate the effect of soil characteristics on selected potato tuber minerals composition. Eight sites located in Nyandarua County in Kenya were selected for this study. The study was carried out between April and September 2013. Soil samples were randomly collected from representative portion of the field in each site. All the samples from each site after packing were taken for laboratory analysis for minerals analysis using standard methods. Well sprouted diffused light stored (DLS) seeds and fresh potato seeds from four varieties (Kenya Mpya, Dutch Robjin, Tigoni and Cangi) were grown in four sites under standard conditions. After harvesting, ten mature tubers of each variety per site and seed potato storage were packed in net bags and taken for minerals evaluation. Data was analyzed using statistical system version 9.

Soil minerals differed significantly $(\mathrm{p} \leq 0.05)$ with sites. Generally, potato tuber minerals significantly $(\mathrm{p} \leq 0.05)$ differed with sites, potato varieties and seed potato storage (fresh or eight-month DLS seed). There was a positive correlation between potato tuber minerals levels and the soil minerals content. Potato minerals were thus affected by potato variety, seed potato storage and soil characteristics in a production site.

DLS seed storage should be adopted so as to produce improved nutritious potatoes for processing. Soil and potato tuber analyses should be extended to other potato growing areas and should be conducted regularly after every two years for potatoes nutritional improvement.
\end{abstract}

Keywords: plant locality, variety, diffused light storage, soil minerals, potato tuber minerals

\section{Introduction}

The potato Solanum tuberosum is a greatly shortened and swollen part of an underground stem commonly grown as starchy tubers (Ekin, 2011). The potato crop is grown in most highland areas (1800-3000 m.a.s.l) like Nyandarua County where it performs better in terms of yield in comparison to other staple foods such as maize (MoA, 2005). It is a short season crop maturing between three to four months and as such, it is grown twice annually and sometimes even thrice depending on the variety and weather conditions among other factors. The potatoes being rich in essential minerals provide both biological and nutritional value to human beings (Ekin, 2011).

Potatoes grow well in friable well-drained aerated and porous soils with $\mathrm{pH}$ close to 5.6 while containing essential nutrients in the right proportions for the following macronutrients: nitrogen $(\mathrm{N})$, phosphorus $(\mathrm{P})$, potassium $(\mathrm{K})$, calcium $(\mathrm{Ca})$, magnesium $(\mathrm{Mg})$, sulphur $(\mathrm{S})$ and carbon $(\mathrm{C})$ and micronutrients such as: iron $(\mathrm{Fe})$, manganese $(\mathrm{Mn})$, zinc $(\mathrm{Zn})$ and copper $(\mathrm{Cu})$ (Singer \& Munns, 1987). Potato requires rainfall in the range 500-700mm during the growth period of 100-150 days (Dent \& Young, 1993). Acid soils are likely to be deficient in $\mathrm{Ca}, \mathrm{Mg}$ and $\mathrm{K}$. Strongly and very strongly acid soils make Al, Fe and $\mathrm{Mn}$ to exist in toxic amounts due to their increased solubility and will cause phosphates to react with these minerals to form insoluble phosphates through fixation leading to deficiency of $\mathrm{P}$ for the plant utilization. The alkaline soils on the other hand make Fe, Mn, $\mathrm{Zn}$ and $\mathrm{Cu}$ to become unavailable for potato growth (Jackson, 1956; Tan, 2005; Kanyanjua \& Agaya, 2006). Soils with adequate nutrients enable potatoes to grow well making tubers to acquire essential minerals (especially N, P and K) for human nutritional quality without artificial fertilization (MoA, 2005). There is lack of adequate information on the status of the soil characteristics and the potato tuber minerals in the potato 
production areas in Nyandarua County.

The objective of the present study was to determine the effect of soil characteristics on potato tuber mineral composition in selected potato growing areas in Nyandarua County.

\section{Materials and Methods}

\subsection{The Study Sites}

The National Potato Research Centre, KARI-Tigoni (considered as a control station), Njabini Sub Center, Pyhort-a farmers'group, Kagema-a farmers'group, Hellen-an individual farmer, Evergreen a farmers' group, Jane-an individual farmer and Gitahi-an individual farmer were chosen as study sites (Table 1). These farmers were purposely selected by respective District Agricultural Officers (DAO) based on their adequate knowledge and skills in potato production. This study was carried out between April and September 2013. The experimental design used was completely randomised block design (CRBD), where eight growth sites were considered. The independent factors considered were: production sites, potato seed storage and potato varieties while dependent factors were: soil and potato tubers minerals, soil $\mathrm{pH}$, soil organic carbon and soil total nitrogen.

Table 1. Summary of study sites

\begin{tabular}{llllll}
\hline Site & Sub county & County & Altitude (m.a.s.l) & Latitude (South) & Longitude (East) \\
\hline Pyhort & Ol-joro-ok & Nyandarua & 2672.791 & $0^{\circ}, 5.057^{\prime}$ & $36^{\circ}, 17.183^{\prime}$ \\
Kagema & Ol-joro-ok & Nyandarua & 2685.897 & $0^{\circ}, 5.122^{\prime}$ & $36^{\circ}, 16.969^{\prime}$ \\
Evergreen & Ol Kalau & Nyandarua & 2520.391 & $0^{\circ}, 18.666^{\prime}$ & $36^{\circ}, 19.657^{\prime}$ \\
Hellen & Kipipiri Githioro & Nyandarua & 2228.697 & $0^{\circ}, 27.719^{\prime}$ & $36^{\circ}, 29.659^{\prime}$ \\
Jane & Kipipiri-Manunga & Nyandarua & 2254.605 & $0^{\circ}, 22.134^{\prime}$ & $36^{\circ}, 29.621^{\prime}$ \\
Gitahi & Mkungi-Engineer & Nyandarua & 2934.614 & $0^{\circ}, 31.01^{\prime}$ & $36^{\circ}, 33.271^{\prime}$ \\
Njabini s/center & Njabini & Nyandarua & 2544.775 & $0^{\circ}, 44.159^{\prime}$ & $36^{\circ}, 38.996^{\prime}$ \\
Tigoni KARI & Limuru & Kiambu & 2131.000 & $1^{\circ}, 15^{\prime}$ & $23^{\circ}, 46^{\prime}$ \\
\hline
\end{tabular}

\subsection{Soil Sampling and Analysis}

Random sampling method was used where individual soil samples were randomly collected across the representative portion of the field using a zigzag soil sampling method according to Carter and Gregorich (2008). The sampling was carried out manually by a hoe $0-25 \mathrm{~cm}$ depth where fifteen soil cores were taken, bulked, mixed thoroughly and $250 \mathrm{~g}$ were packed in polythene paper bags. All the packed samples from each site were taken for laboratory analysis.

Soil preparation involved air drying at $35{ }^{\circ} \mathrm{C}$ for a day followed by grinding and sieving with a $2 \mathrm{~mm}$ sieve according to Tan (2005).

Soil $\mathrm{pH}$ was determined in water in the ratio 1:1 (soil: water respectively) with ELL glass electrode $\mathrm{pH}$ meter according to Maclean (1965) and Walingo et al. (1989). Organic carbon was determined by calorimetric method by oxidizing the soil organic carbon by acidified dichromate at $150{ }^{\circ} \mathrm{C}$ for 30 minutes, cooled by addition of barium chloride, mixed thoroughly, left to stand overnight and it was read on the spectrophotometer at $600 \mathrm{~nm}$ according to Walingo et al. (1989). Total nitrogen was determined by micro-Kjeldahl method according to AOAC (1980). Exchangeable cations (Magnesium, sodium, potassium, manganese, phosphorus and calcium) were determined according to Mehlich Double Acid method (Mehlich, 1959) as outlined in Walingo et al. (1989). Iron, zinc and copper were determined by first being extracted in the oven-dry soil samples in 1:10 ratio (w/v) with $0.1 \mathrm{M} \mathrm{HCL}$ and then determined with atomic absorption spectrophotometer as outlined in Walingo et al. (1989) and AOAC, (1980).

\subsection{Collection of Rainfall Data}

Rainfall data was obtained from the Kenya Meteorological Department offices in the various districts where the study was carried out (Table 2). 
Table 2. Total rainfall $(\mathrm{mm})$ in sub-counties of study sites

\begin{tabular}{lllll}
\hline Month 2013 & Kagema, Pyhort and Evergreen & Jane and Hellen & Njabini and Gitahi & KARI Tigoni \\
\hline March & 124.6 & 107 & 243.1 & 124.6 \\
April & 295.1 & 227 & 553.7 & 295.1 \\
May & 106.7 & 189 & 150.7 & 106.7 \\
June & 98.5 & 52.5 & 79.6 & 98.5 \\
July & 214.1 & 91.2 & 49.8 & 214.1 \\
August & 229.4 & 108 & 78.1 & 229.4 \\
September & 66.8 & 72 & 304.4 & 66.8 \\
Total rainfall & 1135.2 & 846.7 & 1459.4 & 1125.2 \\
\hline
\end{tabular}

Source: Kenya Meteorological Department, 2013.

The rainfall in the study areas during the potato growth period of 100-150 days was adequate since all sites had rainfall in the range 500-700 mm which is sufficient according to Dent and Young (1993), and Muthoni and Kabira (2010).

\subsection{Potato Tuber Analysis}

\subsubsection{Sample Preparation}

Only four potato sites: The National Potato Research Centre KARI-Tigoni (considered as a control station), Pyhort-a farmers' group, Kagema-a farmers' group and Hellen-an individual farmer were considered because the rest had inconsistent potato varieties. Potato seeds for four varieties (Kenya Mpya, Dutch Robjin, Tigoni and Cangi) which had been stored under diffused light storage (DLS) for eight months in farmers' well ventilated stores and freshly harvested potato seeds were planted under standard cultural conditions in the above localities (Lung'hao \& Kabira, 1999). Tubers were manually harvested upon maturity and ten potato tubers about $>35 \mathrm{~mm}$ long from each variety, plant location and seed storage were randomly sorted, packed in net bags and transported to NARL-KARI for minerals analysis. They were then washed, rinsed in deionized water and briefly air-dried.

Since mineral concentrations can vary between the stem end and the distal end of the potato tuber and some cations such as $\mathrm{K}, \mathrm{Ca}, \mathrm{Mg}, \mathrm{Fe}, \mathrm{Zn}, \mathrm{Mn}$ and $\mathrm{Cu}$ are more concentrated in the potato skin relative to the flesh (Karenlampi \& White, 2009), potato tubers were analyzed without peeling. Whole unpeeled tubers were sliced, oven dried at 105 ${ }^{\circ} \mathrm{C}$ until there was no further loss in weight and mixed before analysis.

\subsubsection{Determination of Potato Tubers' $\mathrm{Ca}, \mathrm{Mg}, \mathrm{Cu}, \mathrm{Zn}, \mathrm{Mn}, \mathrm{Fe}, \mathrm{P}$ and $\mathrm{K}$}

The above dried potato samples were first oxidized by hydrogen peroxide (30\%) at a relatively low temperature $\left(100{ }^{\circ} \mathrm{C}\right)$. After decomposition of excess peroxide and evaporation of water, the digestion was completed by concentrated sulphuric acid at elevated temperature $\left(330^{\circ} \mathrm{C}\right)$ in selenium catalyst. Then $\mathrm{Ca}, \mathrm{Mg}, \mathrm{Cu}, \mathrm{Zn}, \mathrm{Mn}$ and Fe were determined with atomic absorption spectrophotometer; $\mathrm{K}$ was determined with a flame photometer; $\mathrm{P}$ was determined calorimetrically on spectrophotometer according to Walingo et al. (1989) and AOAC (1980).

\subsection{Statistical Analysis}

Analysis of variance (ANOVA) and least significant difference test for the soil and potato tubers minerals were conducted using Statistical Analysis System (SAS version 9). Pearson correlation analysis was also performed to determine linear relationships where necessary. Differences $\mathrm{p} \leq 0.05$ were considered significant.

\section{Results and Discussion}

\subsection{Soil Assessment}

The soil $\mathrm{pH}$ in the study sites ranged from 4.0 to 5.72 (Table 3). All the sites had moderately acid soils except KARI- Tigoni and Pyhort which had strongly and very strongly acid soils, respectively (Jackson, 1956). This could be due to acidic parent rock and continuous application of DAP fertilizer (18N: $46 \mathrm{P}_{2} \mathrm{O}_{5}$ ) (Kiiya et al., 2006; Muthoni \& Kabira, 2010). Gitahi's farm had satisfactory soil $\mathrm{pH}$ since it was 5.72 this was $>5.5$ recommended for potato production (Kanyanjua \& Agaya, 2006). This high $\mathrm{pH}$ could be attributed to incorporation of proper rotation regimes with legumes and addition of farm manure. Soil $\mathrm{pH}$ values were significantly $(\mathrm{p} \leq 0.05)$ different in potato plant localities except in the soils at Jane's and Hellen's due to having originated from 
different and similar parent rocks respectively (Recke et al., 1997). This high acidity in the affected soils may lead to deficiencies in essential minerals like $\mathrm{P}, \mathrm{Ca}, \mathrm{Mg}$ and $\mathrm{K}$ while causing $\mathrm{Al}, \mathrm{Fe}$ and $\mathrm{Mn}$ to exist in toxic amounts due to their increased solubility. Excess $\mathrm{Al}, \mathrm{Fe}$ and $\mathrm{Mn}$ react with phosphates to form insoluble phosphates through fixation leading to P deficiency (Jackson, 1956; Tan, 2005).

Soil organic carbon in the study areas significantly $(\mathrm{p} \leq 0.05)$ differed with study area ranging from 1.15 to $4.23 \%$. Organic carbon level was low at Gitahi, this could be due to continuous removal of crop residues for feeding livestock having negative implication on the potatoes growth and quality (Muthoni \& Kabira, 2010).

Table 3. Soil fertility in terms of $\mathrm{pH}$ values, \% Organic Carbon and \% Total Nitrogen

\begin{tabular}{lllllll}
\hline Site & $\mathrm{pH}$ & Soil value & $\%$ Org. carbon & Adequacy & $\% \mathrm{~N}$ & Adequacy \\
\hline Pyhort & $4.0 \pm 0 \mathrm{~g}$ & V. strongly acid & $2.69 \pm 0.01 \mathrm{j}$ & Adequate & $0.27 \pm 0.01 \mathrm{t}$ & Adequate \\
Kagema & $5.2 \pm 0.1 \mathrm{c}$ & Moderately acid & $4.23 \pm 0.02 \mathrm{~h}$ & Adequate & $0.42 \pm 0.0 \mathrm{r}$ & Adequate \\
Evergreen & $5.3 \pm 0.01 \mathrm{~b}$ & Moderately acid & $2.04 \pm 0.0 \mathrm{k}$ & Moderate & $0.21 \pm 0.01 \mathrm{u}$ & Adequate \\
Hellen & $5.04 \pm 0 \mathrm{~d}$ & Moderately acid & $1.79 \pm 0.01 \mathrm{n}$ & Moderate & $0.18 \pm 0.02 \mathrm{w}$ & Low \\
Jane & $5.04 \pm 0 \mathrm{~d}$ & Moderately acid & $1.59 \pm 0.0 \mathrm{p}$ & Moderate & $0.16 \pm 0.01 \mathrm{x}$ & Low \\
Gitahi & $5.72 \pm 0.01 \mathrm{a}$ & Moderately acid & $1.15 \pm 0.02 \mathrm{q}$ & Low & $0.12 \pm 0.0 \mathrm{y}$ & Low \\
Njabini & $5.03 \pm 0.02 \mathrm{e}$ & Moderately acid & $2.88 \pm 0.0 \mathrm{i}$ & Adequate & $0.29 \pm 0.01 \mathrm{~s}$ & Adequate \\
KARI Tigoni & $4.57 \pm 0.01 \mathrm{f}$ & Strongly acid & $1.92 \pm 0.1 \mathrm{~m}$ & Moderate & $0.19 \pm 0.01 \mathrm{v}$ & Low
\end{tabular}

Results are means of two determinations. Means with the same letter in the same column are not significantly (p $\leq 0.05$ ) different.

Soil nitrogen in the study sites significantly ( $\mathrm{p} \leq 0.05$ ) differed ranging from 0.12 to $0.42 \%$. Total nitrogen was low at KARI-Tigoni, Hellen, Jane and Gitahi probably due to failure to incorporate legumes in the rotation cycle in potato farms affecting potatoes growth and tuber quality.

Soil phosphorus was $10 \mathrm{ppm}$ and $30 \mathrm{ppm}$ at Hellen and Njabini sub/center respectively (Table 4). Phosphorus, being a macronutrient, its content in the soils was generally low. This was probably due to high soil acidity found in the sites. Despite the fact that DAP fertilizer is being applied continuously in potato planting areas, P content in the soil was low and this could be attributed to the low $\mathrm{pH}$ and lack of organic manure application. The soil phosphorus significantly differed $(\mathrm{p} \leq 0.05)$ in potato production sites possibly due to the soil parent rock differences (Recke et al, 1997; Muthoni and Kabira, 2010). Phosphorus deficiency causes potato plants to produce tubers with lower specific gravity compared to those with adequate phosphorus nutrition (Woolfe, 1987).

Table 4. Minerals in the study sites

\begin{tabular}{llllllllll}
\hline Site & $\mathrm{P}(\mathrm{ppm})$ & $\mathrm{Fe}(\mathrm{ppm})$ & $\mathrm{Zn}(\mathrm{ppm})$ & $\mathrm{K}(\mathrm{me})$ & $\mathrm{Ca}(\mathrm{me})$ & $\mathrm{Mg}(\mathrm{me})$ & $\mathrm{Mn}(\mathrm{me})$ & $\mathrm{Cu}(\mathrm{ppm})$ & $\mathrm{Na}(\mathrm{me})$ \\
\hline Pyhort & $20 \pm 0.03 \mathrm{~d}$ & $144 \pm 0.01 \mathrm{~b}$ & $5.33 \pm 0.01 \mathrm{~h}$ & $0.68 \pm 0.01 \mathrm{~d}$ & $2.1 \pm 0.02 \mathrm{f}$ & $2.83 \pm 0.01 \mathrm{~d}$ & $0.7 \pm 0.01 \mathrm{e}$ & $1.97 \pm 0.01 \mathrm{~d}$ & $0.22 \pm 0.01 \mathrm{e}$ \\
Kagema & $15 \pm 0.01 \mathrm{e}$ & $127 \pm 0.01 \mathrm{c}$ & $31.3 \pm 0.22 \mathrm{~b}$ & $0.88 \pm 0.03 \mathrm{~b}$ & $5.7 \pm 0.01 \mathrm{a}$ & $3.61 \pm 0.02 \mathrm{a}$ & $1.17 \pm 0.01 \mathrm{a}$ & $1.72 \pm 0.01 \mathrm{e}$ & $0.42 \pm 0.01 \mathrm{~b}$ \\
Hellen & $10 \pm 0.06 \mathrm{f}$ & $97.1 \pm 0.14 \mathrm{~d}$ & $7.44 \pm 0.02 \mathrm{~d}$ & $0.9 \pm 0.02 \mathrm{~b}$ & $2.9 \pm 0.07 \mathrm{~d}$ & $3.37 \pm 0.01 \mathrm{~b}$ & $0.9 \pm 0.02 \mathrm{c}$ & $11.7 \pm 0.07 \mathrm{a}$ & $0.26 \pm 0.01 \mathrm{~d}$ \\
Njabini s/center & $30 \pm 0.06 \mathrm{a}$ & $88.9 \pm 0.07 \mathrm{e}$ & $40.2 \pm 0.01 \mathrm{a}$ & $0.64 \pm 0.01 \mathrm{de}$ & $2.7 \pm 0.02 \mathrm{e}$ & $3.36 \pm 0.01 \mathrm{~b}$ & $0.9 \pm 0.01 \mathrm{c}$ & $2.96 \pm 0.01 \mathrm{c}$ & $0.22 \pm 0.01 \mathrm{e}$ \\
Tigoni KARI & $20 \pm 0.08 \mathrm{~d}$ & $81.4 \pm 0.14 \mathrm{f}$ & $28.5 \pm 0.04 \mathrm{c}$ & $1.16 \pm 0.02 \mathrm{a}$ & $2.9 \pm 0.01 \mathrm{~d}$ & $0.9 \pm 0.07 \mathrm{e}$ & $0.75 \pm 0.01 \mathrm{~d}$ & $4 \pm 0.02 \mathrm{~b}$ & $0.24 \pm 0.01 \mathrm{e}$ \\
Gitahi & $25 \pm 0.67 \mathrm{c}$ & $46.4 \pm 0.67 \mathrm{~g}$ & $5.76 \pm 0.03 \mathrm{~g}$ & $1.14 \pm 0.01 \mathrm{a}$ & $3.3 \pm 0.07 \mathrm{c}$ & $3.4 \pm 0.01 \mathrm{~b}$ & $0.65 \pm 0.01 \mathrm{f}$ & $1.11 \pm 0.02 \mathrm{~g}$ & $1.27 \pm 0.01 \mathrm{a}$ \\
Jane & $20 \pm 0.04 \mathrm{~d}$ & $19 \pm 0.28 \mathrm{~h}$ & $6.03 \pm 0.02 \mathrm{f}$ & $0.64 \pm 0.01 \mathrm{e}$ & $3.9 \pm 0.07 \mathrm{~b}$ & $3.39 \pm 0.01 \mathrm{~b}$ & $1.01 \pm 0.01 \mathrm{~b}$ & $2.01 \pm 0.01 \mathrm{~d}$ & $0.36 \pm 0.01 \mathrm{c}$ \\
Evergreen & $25 \pm 0.01 \mathrm{~b}$ & $179 \pm 0.14 \mathrm{a}$ & $7.27 \pm 0.02 \mathrm{e}$ & $0.78 \pm 0.01 \mathrm{c}$ & $2.9 \pm 0.01 \mathrm{~d}$ & $2.9 \pm 0.04 \mathrm{c}$ & $0.63 \pm 0.01 \mathrm{~g}$ & $1.36 \pm 0.01 \mathrm{f}$ & $0.22 \pm 0.01 \mathrm{ef}$ \\
\hline
\end{tabular}

Results are means of two determinations with \pm standard deviation. Means with the same letter in the same column are not significantly $(\mathrm{p} \leq 0.05)$ different. 
Soil iron ranged from $19.0 \mathrm{ppm}$ at Jane's to $179 \mathrm{ppm}$ at Evergreen (Table 4). The soil iron content significantly $(\mathrm{p} \leq 0.05)$ differed in all the sites due to parent rock but it was adequate for the potato growth (Recke et al., 1997).

Calcium content in the soil varied from $2.1 \mathrm{me}$ at Pyhort to 5.7me at Kagema. Soil Ca differed (p $\leq 0.05)$ significantly in all the sites due to differences in the parent rock (Singer and Munns, 1987). However Ca, a macronutrient was adequate for the potato growth in all the sites (Recke et al., 1997).

Soil magnesium as ranged from 0.9 me at Tigoni-KARI to $3.61 \mathrm{me}$ at Kagema (Table4). It significantly $(\mathrm{p} \leq 0.05)$ differed in all the sites and it occurred in high / toxic levels due to continuous application of DAP fertilizer (Kanyanjua \& Agaya, 2006; Recke et al., 1997).

Soil manganese ranged from 0.6 at Evergreen to 1.17 at Kagema; Soil copper ranged from 1.11 ppm at Gitahi to $11.7 \mathrm{ppm}$ at Hellen and soil sodium ranged from $0.22 \mathrm{me}$ at Pyhort to $1.27 \mathrm{me}$ at Gitahi (Table4). All these minerals significantly $(\mathrm{p} \leq 0.05)$ differed in all the sites due to differences in the parent rock. However they were generally adequate in the sites (Okalebo, 1985; Kanyanjua \& Agaya, 2006; Recke et al., 1997).

There was significant $(\mathrm{P} \leq 0.05)$ correlation $(\mathrm{r}=0.17)$ between $\mathrm{pH}$ and phosphorus. There was also significant $(\mathrm{P}>0.05)$ correlation $(\mathrm{r}=0.32)$ between $\mathrm{pH}$ and potassium (Table 5). The positive correlations demonstrate that the higher the $\mathrm{pH}$ (less acidity) the higher the minerals content and vice versa.

Table 5. Pearson correlation coefficient (r) between $\mathrm{pH}$ and levels of phosphorus; $\mathrm{pH}$ and Potassium

\begin{tabular}{llll}
\hline Parameters & $\mathrm{pH}$ & Phosphorus & Potassium \\
\hline $\mathrm{pH}$ & 1.00 & $0.17^{\mathrm{a}}$ & $0.32^{\mathrm{a}}$ \\
Phosphorus & $0.17^{\mathrm{a}}$ & 1.00 & \\
Potassium & $0.32^{\mathrm{a}}$ & & 1.00
\end{tabular}

${ }^{\mathrm{a}}$ Significant correlation coefficient $(\mathrm{P} \leq 0.05)(\mathrm{N}=32)$.

Potato farmers in Nyandarua County having intensively cultivated their small farms with potatoes without proper crop rotation, coupled with extensive use of acidifying fertilizer (DAP) have contributed to reduction in the soil minerals (Muthoni \& Kabira, 2010).

\subsection{Results on Potato Tubers Minerals}

Phosphorus content in potato tubers ranged from $315 \mathrm{mg} / 100 \mathrm{~g}$ in Kenya Mpya seed stored for 8 months under DLS in Kagema to $495 \mathrm{mg} / 100 \mathrm{~g}$ in unstored Cangi seed in Pyhort. Phosphorus significantly differed $(\mathrm{p} \leq 0.05)$ with production sites, potato varieties and storage of the seed potato (fresh or eight-month DLS seed) and with interactions among production site, variety and seed storage (Table 6). Order of P level according to site was as follows: Pyhort $<$ Hellen $<$ Tigoni-KARI $<$ Kagema. Potato varieties differed in P content due to their differences in their genetic make-up and soil P (Table 4) this in agreement with Tan (2005). It is very important to the essential process of metabolism in the body. Phosphorus is a constituent of the nucleoproteins (Wiley, 2006). 
Table 6. Mineral contents (mg/100g DWB) of four potato varieties basing on plant locality and potato seed treatment

\begin{tabular}{|c|c|c|c|c|c|c|c|c|c|c|}
\hline Site & Variety & Treat & $\mathrm{P}$ & $\mathrm{Fe}$ & $\mathrm{Zn}$ & $\mathrm{K}$ & $\mathrm{Ca}$ & $\mathrm{Mg}$ & $\mathrm{Mn}$ & $\mathrm{Cu}$ \\
\hline \multirow[t]{8}{*}{ Hellen } & Cangi & Fresh & $385 \pm 0.01 \mathrm{~g}$ & $61.55 \pm 0.11 \mathrm{a}$ & $2.305 \pm 0.07 p$ & $1825 \pm 0.02 \mathrm{j}$ & $100 \pm 0.014 \mathrm{~b}$ & $135 \pm 0.01 \mathrm{c}$ & $4.11 \pm 0.14 \mathrm{~d}$ & $0.75 \pm 0.01 \mathrm{c}$ \\
\hline & & Stored & $385 \pm 0.01 \mathrm{~g}$ & $61.55 \pm 0.11 \mathrm{a}$ & $2.305 \pm 0.07 \mathrm{p}$ & $1825 \pm 0.02 \mathrm{j}$ & $100 \pm 0.014 \mathrm{~b}$ & $135 \pm 0.01 \mathrm{c}$ & $4.11 \pm 0.14 \mathrm{~d}$ & $0.75 \pm 0.01 \mathrm{c}$ \\
\hline & Dutch & Fresh & $445 \pm 0.01 \mathrm{~d}$ & $15.95 \pm 0.11 \mathrm{~s}$ & $2.235 \pm 0.07 \mathrm{q}$ & $1445 \pm 0.01 \mathrm{r}$ & $215 \pm 0.002 \mathrm{a}$ & $125 \pm 0.01 \mathrm{~d}$ & $2.375 \pm 0.07 \mathrm{q}$ & $0.118 \pm 0.01 \mathrm{zc}$ \\
\hline & & Stored & $465 \pm 0.01 \mathrm{~b}$ & $18.05 \pm 0.11 \mathrm{n}$ & $2.505 \pm 0.07 \mathrm{~h}$ & $1296 \pm 0.01 \mathrm{w}$ & $10 \pm 0.000 \mathrm{p}$ & $800 \pm 0.00 \mathrm{a}$ & $2.605 \pm 0.07 \mathrm{n}$ & $0.67 \pm 0.14 \mathrm{~d}$ \\
\hline & KMpya & Fresh & $325 \pm 0.01 \mathrm{q}$ & $15.65 \pm 0.11 \mathrm{t}$ & $1.775 \pm 0.07 \mathrm{za}$ & $1297 \pm 0.01 \mathrm{v}$ & $2 \pm 0.000 \mathrm{~s}$ & $75 \pm 0.01 \mathrm{~m}$ & $1.975 \pm 0.07 \mathrm{v}$ & $0.600 \pm 0.01 \mathrm{~h}$ \\
\hline & & Stored & $355 \pm 0.011$ & $14.95 \pm 0.11 \mathrm{w}$ & $1.855 \pm 0.07 \mathrm{x}$ & $1460 \pm 0.01 \mathrm{q}$ & $11 \pm 0.001 \mathrm{n}$ & $61.5 \pm 0.00 \mathrm{p}$ & $1.905 \pm 0.07 \mathrm{w}$ & $0.601 \pm 0.01 \mathrm{~g}$ \\
\hline & Tigoni & Fresh & $315 \pm 0.01 \mathrm{t}$ & $27.15 \pm 0.11 \mathrm{f}$ & $2.05 \pm 0.56 \mathrm{u}$ & $2660 \pm 0.07 \mathrm{a}$ & $30 \pm 0.000 \mathrm{e}$ & $70 \pm 0.00 \mathrm{n}$ & $2.585 \pm 0.07 \mathrm{k}$ & $0.543 \pm 0.01 \mathrm{j}$ \\
\hline & & Stored & $335 \pm 0.01 \mathrm{q}$ & $30.95 \pm 0.11 \mathrm{e}$ & $2.745 \pm 0.64 \mathrm{e}$ & $2075 \pm 0.04 \mathrm{f}$ & $20 \pm 0.000 \mathrm{~h}$ & $85 \pm 0.01 \mathrm{j}$ & $2.675 \pm 0.07 \mathrm{f}$ & $0.651 \pm 0.00 \mathrm{e}$ \\
\hline \multirow[t]{8}{*}{ Kagema } & Cangi & Fresh & $355 \pm 0.011$ & $19.8 \pm 1.41 \mathrm{~m}$ & $2.31 \pm 0.14 \mathrm{n}$ & $1750 \pm 0.01 \mathrm{k}$ & $20.5 \pm 0.001 \mathrm{~g}$ & $37.5 \pm 0.00 \mathrm{t}$ & $2.47 \pm 0.14 p$ & $0.455 \pm 0.07 \mathrm{p}$ \\
\hline & & Stored & $355 \pm 0.011$ & $19.8 \pm 1.41 \mathrm{~m}$ & $2.31 \pm 0.14 \mathrm{n}$ & $1750 \pm 0.01 \mathrm{k}$ & $20.5 \pm 0.001 \mathrm{~g}$ & $37.5 \pm 0.00 \mathrm{t}$ & $2.47 \pm 0.14 p$ & $0.455 \pm 0.07 \mathrm{p}$ \\
\hline & Dutch & Fresh & $325 \pm 0.01 \mathrm{r}$ & $13.35 \pm 0.71 \mathrm{yt}$ & $1.74 \pm 0.14 \mathrm{za}$ & $1605 \pm 0.01 \mathrm{p}$ & $10 \pm 0.000 \mathrm{p}$ & $90 \pm 0.00 \mathrm{i}$ & $1.555 \pm 0.35 \mathrm{x}$ & $0.134 \pm 0.01 \mathrm{zb}$ \\
\hline & & Stored & $455 \pm 0.01 \mathrm{c}$ & $35.95 \pm 0.71 \mathrm{c}$ & $6.25 \pm 0.71 b$ & $2250 \pm 0.01 \mathrm{~d}$ & $20 \pm 0.000 \mathrm{~h}$ & $85 \pm 0.01 \mathrm{j}$ & $2.055 \pm 0.07 \mathrm{v}$ & $0.812 \pm 0.01 \mathrm{a}$ \\
\hline & KMpya & Fresh & $325 \pm 0.01 \mathrm{r}$ & $14.55 \pm 0.71 \mathrm{wx}$ & $1.905 \pm 0.07 \mathrm{w}$ & $2335 \pm 0.01 \mathrm{c}$ & $3 \pm 0.000 \mathrm{r}$ & $95 \pm 0.01 \mathrm{~h}$ & $1.112 \pm 0.13 \mathrm{y}$ & $0.445 \pm 0.07 \mathrm{q}$ \\
\hline & & Stored & $315 \pm 0.01 \mathrm{t}$ & $16.55 \pm 0.71 \mathrm{p}$ & $1.75 \pm 0.14 \mathrm{zb}$ & $1655 \pm 0.04 \mathrm{~m}$ & $10 \pm 0.000 \mathrm{p}$ & $470 \pm 0.54 b$ & $2.215 \pm 0.07 \mathrm{u}$ & $0.184 \pm 0.01 \mathrm{z}$ \\
\hline & Tigoni & Fresh & $340 \pm 0.00 p$ & $20.35 \pm 0.71 \mathrm{k}$ & $1.965 \pm 0.07 \mathrm{v}$ & $2035 \pm 0.01 \mathrm{~g}$ & $19 \pm 0.001 \mathrm{j}$ & $120 \pm 0.01 \mathrm{e}$ & $2.245 \pm 0.07 \mathrm{t}$ & $0.368 \pm 0.01 \mathrm{u}$ \\
\hline & & Stored & $375 \pm 0.01 \mathrm{~h}$ & $31.15 \pm 0.71 \mathrm{~d}$ & $2.62 \pm 0.14 \mathrm{~g}$ & $1875 \pm 0.01 \mathrm{i}$ & $10 \pm 0.000 \mathrm{p}$ & $125 \pm 0.01 \mathrm{~d}$ & $2.215 \pm 0.07 \mathrm{u}$ & $0.784 \pm 0.01 \mathrm{~b}$ \\
\hline \multirow[t]{8}{*}{ Pyhort } & Cangi & Fresh & $495 \pm 0.01 \mathrm{a}$ & $21.65 \pm 0.71 \mathrm{i}$ & $2.505 \pm 0.07 \mathrm{~h}$ & $214 \pm 0.01 \mathrm{za}$ & $9.5 \pm 0.001 \mathrm{q}$ & $85 \pm 0.01 \mathrm{j}$ & $3.07 \pm 0.00 \mathrm{f}$ & $0.550 \pm 0.01 \mathrm{i}$ \\
\hline & & Stored & $495 \pm 0.01 \mathrm{a}$ & $21.65 \pm 0.71 \mathrm{i}$ & $2.505 \pm 0.07 \mathrm{~h}$ & $214 \pm 0.01 \mathrm{za}$ & $9.5 \pm 0.001 \mathrm{q}$ & $85 \pm 0.01 \mathrm{j}$ & $3.07 \pm 0.14 \mathrm{f}$ & $0.550 \pm 0.01 \mathrm{i}$ \\
\hline & Dutch & Fresh & $375 \pm 0.01 \mathrm{i}$ & $36.55 \pm 0.71 \mathrm{~b}$ & $3.26 \pm 0.14 \mathrm{c}$ & $1445 \pm 0.01 \mathrm{r}$ & $29.5 \pm 0.001 \mathrm{f}$ & $80.5 \pm 0.01 \mathrm{jk}$ & $2.365 \pm 0.07 \mathrm{r}$ & $0.649 \pm 0.01 \mathrm{f}$ \\
\hline & & Stored & $385 \pm 0.01 \mathrm{~g}$ & $36.05 \pm 0.71 \mathrm{bc}$ & $7.815 \pm 0.07 \mathrm{a}$ & $1425 \pm 0.01 \mathrm{~s}$ & $50.5 \pm 0.001 \mathrm{c}$ & $95 \pm 0.01 \mathrm{~h}$ & $2.625 \pm 0.07 \mathrm{i}$ & $0.136 \pm 0.07 \mathrm{za}$ \\
\hline & KMpya & Fresh & $345 \pm 0.01 \mathrm{n}$ & $23.85 \pm 0.71 \mathrm{~h}$ & $1.81 \pm 0.14 \mathrm{y}$ & $1325 \pm 0.01 \mathrm{t}$ & $2 \pm 0.000 \mathrm{~s}$ & $38 \pm 0.01 \mathrm{~s}$ & $2.125 \pm 0.07$ ua & $0.531 \pm 0.02 \mathrm{k}$ \\
\hline & & Stored & $395 \pm 0.01 \mathrm{e}$ & $21.15 \pm 0.71 \mathrm{j}$ & $2.185 \pm 0.07 \mathrm{~s}$ & $1625 \pm 0.02 \mathrm{n}$ & $12.5 \pm 0.004 \mathrm{k}$ & $80 \pm 0.00 \mathrm{k}$ & $2.655 \pm 0.07 \mathrm{~g}$ & $0.367 \pm 0.01 \mathrm{v}$ \\
\hline & Tigoni & Fresh & $385 \pm 0.01 \mathrm{~g}$ & $16.6 \pm 1.41 \mathrm{q}$ & $1.845 \pm 0.21 x y$ & $1305 \pm 0.01 \mathrm{u}$ & $3 \pm 0.000 \mathrm{r}$ & $60 \pm 0.00 \mathrm{r}$ & $2.3 \pm 0.14 \mathrm{~s}$ & $0.356 \pm 0.04 \mathrm{w}$ \\
\hline & & Stored & $365 \pm 0.01 \mathrm{k}$ & $16.25 \pm 0.71 \mathrm{r}$ & $2.115 \pm 0.07 \mathrm{t}$ & $1295 \pm 0.01 \mathrm{x}$ & $10.5 \pm 0.001 \mathrm{~m}$ & $69.5 \pm 0.00 \mathrm{p}$ & $2.615 \pm 0.07 \mathrm{j}$ & $0.482 \pm 0.01 \mathrm{~m}$ \\
\hline \multirow[t]{8}{*}{ TigoniKARI } & Cangi & Fresh & $395 \pm 0.01 \mathrm{e}$ & $15.45 \pm 0.71 \mathrm{u}$ & $2.705 \pm 0.07 \mathrm{f}$ & $253 \pm 0.01 y$ & $20.5 \pm 0.001 \mathrm{~g}$ & $100 \pm 0.01 \mathrm{~g}$ & $2.51 \pm 0.21 \mathrm{n}$ & $0.45 \pm 0.71 \mathrm{q}$ \\
\hline & & Stored & $395 \pm 0.01 \mathrm{e}$ & $15.45 \pm 0.71 \mathrm{u}$ & $2.705 \pm 0.07 \mathrm{f}$ & $253 \pm 0.01 \mathrm{y}$ & $20.5 \pm 0.001 \mathrm{~g}$ & $100 \pm 0.01 \mathrm{~g}$ & $2.51 \pm 0.56 n$ & $0.4 \pm 0.00 \mathrm{~s}$ \\
\hline & Dutch & Fresh & $375 \pm 0.01 \mathrm{i}$ & $13.45 \pm 0.71 \mathrm{y}$ & $2.855 \pm 0.07 \mathrm{~d}$ & $224 \pm 0.00 \mathrm{z}$ & $20 \pm 0.000 \mathrm{~h}$ & $120 \pm 0.01 \mathrm{e}$ & $3.835 \pm 0.07 \mathrm{e}$ & $0.416 \pm 0.01 \mathrm{r}$ \\
\hline & & Stored & $370 \pm 0.00 \mathrm{j}$ & $15.25 \pm 0.71 \mathrm{v}$ & $2.335 \pm 0.07 \mathrm{~m}$ & $1885 \pm 0.01 \mathrm{~h}$ & $20 \pm 0.000 \mathrm{~h}$ & $90 \pm 0.00 \mathrm{i}$ & $2.49 \pm 0.14 \mathrm{na}$ & $0.316 \pm 0.01 \mathrm{x}$ \\
\hline & KMpya & Fresh & $335 \pm 0.01 \mathrm{q}$ & $20.75 \pm 0.71 \mathrm{j}$ & $2.245 \pm 0.07 \mathrm{r}$ & $203 \pm 0.01 \mathrm{zb}$ & $2 \pm 0.000 \mathrm{~s}$ & $80 \pm 0.00 \mathrm{k}$ & $7.705 \pm 0.07 \mathrm{~b}$ & $0.475 \pm 0.07 \mathrm{n}$ \\
\hline & & Stored & $350 \pm 0.00 \mathrm{~m}$ & $24.65 \pm 0.71 \mathrm{~g}$ & $2.34 \pm 0.14 \mathrm{k}$ & $2425 \pm 0.01 \mathrm{~b}$ & $10 \pm 0.000 \mathrm{p}$ & $90 \pm 0.00 \mathrm{i}$ & $8.37 \pm 0.14 \mathrm{a}$ & $0.500 \pm 0.01 \mathrm{mn}$ \\
\hline & Tigoni & Fresh & $355 \pm 0.011$ & $11.85 \pm 0.71 \mathrm{z}$ & $2.367 \pm 0.04 \mathrm{i}$ & $2245 \pm 0.01 \mathrm{e}$ & $39 \pm 0.001 \mathrm{~d}$ & $80 \pm 0.00 \mathrm{k}$ & $6.26 \pm 0.42 \mathrm{c}$ & $0.382 \pm 0.01 \mathrm{t}$ \\
\hline & & Stored & $390 \pm 0.00 \mathrm{f}$ & $14.45 \pm 0.71 \mathrm{x}$ & $2.565 \pm 0.21 \mathrm{j}$ & $187 \pm 0.01 \mathrm{zc}$ & $19.5 \pm 0.001 \mathrm{i}$ & $101 \pm 0.00 \mathrm{f}$ & $2.63 \pm 0.14 \mathrm{~h}$ & $0.300 \pm 0.01 \mathrm{y}$ \\
\hline
\end{tabular}

Results are means of two determinations with \pm standard deviation. Means with the same letter in the same column are not significantly $(\mathrm{p}>0.05)$ different.

Iron content in potato tubers ranged from $11.85 \mathrm{mg} / 100 \mathrm{~g}$ dry weight basis (DWB) obtained from fresh planted Tigoni seed in KARI-Tigoni to $61.55 \mathrm{mg} / 100 \mathrm{~g}$ DWB obtained from fresh planted Cangi seed at Hellen's. Iron content significantly $(\mathrm{p} \leq 0.05)$ differed with production site, potato varieties and treatment of the seed potato (fresh or eight-month DLS seed). Interactions among plant locality, variety and seed treatment had significant ( $\mathrm{p} \leq$ 0.05) effect on $\mathrm{Fe}$ content. Although $\mathrm{Fe}$ was within the range given by Burton (1989), it could have been lower if tubers had been peeled. It was generally higher in tubers from DLS seed treatment than in fresh seed. 17-18\% of daily Fe requirements can be provided by a fresh $200 \mathrm{~g}$ potato according to White et al. (2009).

Zinc content in potato tubers ranged from $1.74 \mathrm{mg} / 100 \mathrm{~g}$ DWB obtained from planted fresh Dutch Robjin seed in 
Kagema to $7.815 \mathrm{mg} / 100 \mathrm{~g}$ DWB obtained from planted DLS Dutch Robjin seed in Pyhort. The highest level exceeded the level given by Burton could be because of high acidity. Zinc content significantly $(\mathrm{p} \leq 0.05)$ differed with plant localities, potato varieties and treatment of the seed potato (fresh or eight-month DLS seed). Interactions among plant locality, variety and seed treatment had significant $(\mathrm{p} \leq 0.05)$ effect on Zn content. 5-13 $\%$ of daily $\mathrm{Zn}$ requirements can be provided by a fresh $200 \mathrm{~g}$ potato (White et al., 2009).

Potassium content in potato tubers ranged from $1295 \mathrm{mg} / 100 \mathrm{~g}$ DWB obtained from planted DLS Tigoni seed in Pyhort to $2660 \mathrm{mg} / 100 \mathrm{~g}$ DWB obtained from planted fresh Cangi seed in Tigoni KARI. In a decreasing order, Tigoni KARI had the highest, then Kagema, Hellen and lowest at Pyhort. About 3500mg/day is required by an adult of whom $26.1 \%$ can be obtained from potatoes (Ekin, 2011).

Calcium content in potato tubers ranged from $2 \mathrm{mg} / 100 \mathrm{~g}$ DWB from planted fresh Kenya Mpya seed in Pyhort, Hellen and Tigoni KARI to $100 \mathrm{mg} / 100 \mathrm{~g}$ DWB obtained from fresh Cangi seed in Hellen. According to Burton (1989) this minimum level for Ca was too low to provide any substantial nutritional benefit. Recommended daily $\mathrm{Ca}$ intake for an adult is $1000 \mathrm{mg}$ of which the rich potato can contribute $4.6 \%$.

Magnesium content in potato tubers ranged from $38 \mathrm{mg} / 100 \mathrm{~g}$ DWB obtained from planted fresh Cangi seed in Kagema to $800 \mathrm{mg} / 100 \mathrm{~g}$ DWB obtained from planted DLS Dutch Robjin seed in Hellen which was beyond the level determined by Burton due to its toxic levels in the soil.

Manganese content in potato tubers ranged from $1.112 \mathrm{mg} / 100 \mathrm{~g}$ DWB obtained from planted fresh Kenya Mpya seed in Kagema to $8.37 \mathrm{mg} / 100 \mathrm{~g}$ DWB obtained from planted DLS Kenya Mpya seed in Tigoni KARI and was within the range given by Burton (1989). 5-13\% of daily Mn requirements can be provided by a fresh $200 \mathrm{~g}$ potato (White et al., 2009).

Copper content in potato tubers ranged from $0.118 \mathrm{mg} / 100 \mathrm{~g}$ DWB obtained from planted fresh Dutch Robjin seed in Hellen to $1.355 \mathrm{mg} / 100 \mathrm{~g}$ DWB obtained from planted DLS Dutch Robjin seed in Pyhort was within Burton's range as shown in Burton (1989). Daily copper intake for men and women is $1.2 \mathrm{mg}$ of which 28.3-45.6 $\%$ can be acquired from potato tubers (WHO, 2004).

Generally the minerals $\mathrm{K}, \mathrm{Ca}, \mathrm{Mg}, \mathrm{Mn}$ and $\mathrm{Cu}$ contents significantly $(\mathrm{p} \leq 0.05)$ differed with plant sites, potato varieties and storage of the seed potato (fresh or eight-month DLS seed) and with their interactions. The above minerals varied in according to sites as follows respectively: Pyhort $<$ Hellen $<$ Kagema $<$ Tigoni-KARI; Pyhort/Kagema $<$ Tigoni-KARI $<$ Hellen; Pyhort $<$ Tigoni-KARI $<$ Kagema $<$ Hellen; Kagema $<$ Pyhort $<$ Hellen $<$ Tigoni-KARI; Tigoni-KARI $<$ Kagema $<$ Hellen $<$ Pyhort.

Regularly potato tubers obtained from planted DLS seeds had higher minerals content as compared with potatoes from planted fresh seeds. The potato minerals were affected by potato variety, environmental and soil conditions in a production site and cultural practices including diffused light storage (DLS) seed treatment in agreement with the findings of Tan (2005); Abong' et al. (2011); Ekin (2011).

Table 7. Pearson correlation coefficient (r) between Soil P and Tuber P; Soil K and tuber K

\begin{tabular}{lllll}
\hline Parameters & Soil P & Tuber P & Soil K & Tuber K \\
\hline Soil P & 1.00 & $0.13^{\mathrm{a}}$ & & \\
Tuber P & $0.13^{\mathrm{a}}$ & 1.00 & & \\
Soil K & & & 1.00 & $0.53^{\mathrm{a}}$ \\
Tuber K & & & $0.53^{\mathrm{a}}$ & 1.00 \\
\hline
\end{tabular}

${ }^{\mathrm{a}}$ Significant correlation coefficient $(\mathrm{P} \leq 0.05)(\mathrm{N}=64)$.

Weak significant $(\mathrm{P} \leq 0.05)$ correlations $(\mathrm{r}=0.13)$ between soil phosphorus and tubers phosphorus could be attributed to other prevailing factors such as soil acidity. There existed a significant correlation $(\mathrm{r}=0.53)$ between soil $\mathrm{K}$ and tuber $\mathrm{K}$ (Table 7). The positive correlation in both cases implied direct relationship between soil minerals levels and tubers minerals.

\section{Conclusion and Recommendation}

Soils from all the study sites significantly differed in minerals and $\mathrm{pH}$ levels. The potato minerals were affected by potato variety, soil characteristics in all production sites and the seed potato storage. This implies that potato minerals can be affected by soil characteristics/ level of minerals in a production site. Soil and potato tuber 
analyses should be extended to other potato growing areas in Nyandarua County and in Kenya as a whole and this evaluation should be conducted regularly after every two years.

\section{References}

Abong', G. O., Okoth, M. W., Imungi, J. K., \& Kabira, J. N. (2011). Effects of cultivar, frying temperature and slice thickness on oil uptake and sensory quality of potato crisps. J. Agric. Sci. \&Technol., A.1, 156-163.

Andre C. M., Ghislain, M., Bertin, P. Q., Herrera, M. D. R., Hoffmann, L., ... Evers, D. (2007). Andean potato cultivars (Solanum tuberosum L.) as a source of antioxidant and mineral micronutrients. J. Agric. Food Chem. 55, 366-378. http://dx.doi.org/10.1021/jf062740i

Association of Official Analytical Chemists. (1980). Official Methods of Analysis (13th ed.). AOAC, Washington, DC.

Bowman, A. B., \& Russel, M. R. (2001). Present knowledge in Nutrition (8th ed.). ILSI press Washington, DC.

Burton, W. G. (1989). The potato (3rd ed., pp. 286-360). Longman Group UK, Ltd.

Dent, D., \& Young, A. (1993). Soil Survey and Land Evaluation. E \& FN Spon, an imprint of Chapman \& Hall, London. Glasgow. New York. Tokyo. Melbourne. Madras.

Ekin, Z. (2011). Some analytical quality characteristics for evaluating the utilization and consumption of potato (Solanum tuberosum L.) tubers. African Journal of Biotechnology, 10(32), 6001-6010.

Jackson, M. L. (1958). Soil Chemical Analysis. Eagle Wood Cliff. N. Y. p. 498.

Jaetzold, R., Schmidt, H., Hornetz, B., \& Shisanya, C. (2006). Farm Management Handbook of Kenya (2nd ed., Vol. II, Part B). Natural Conditions and Farm Management Information. Central Kenya. Ministry of Agriculture, Nairobi, Kenya.

Jong, H. D., Sieczka, J. B., \& Jong, W. D. (2011). The Complete Book of Potatoes. What every grower and gardener needs to know. Published by Timber Press, Portland, London.

Kabira, J. N., \& Lemaga, B. (2006). Quality Evaluation procedures for Research and food Industries Applicable in East and Central Africa. A publication of Kenya Agricultural Research Institute, Nairobi, Kenya.

Kaguongo, W. P., Gildemacher, P., Demo, P., Wagoire, W., Kinyae, P., Andrade, J., ... Thiele, G. (2008). Farmer practices and adoption of improved potato varieties in Kenya and Uganda (Social Sciences Working Paper 2008-5). International Potato Center (CIP), Lima, Peru.

Kanyanjua, S. M., \& Agaya, G. O. (2006). A guide to choice of mineral fertilizers in Kenya. KARI Technical Note (No. 17). KARI, Nairobi, Kenya.

Karenlampi, S. O., \& White, P. J. (2009). Potato Proteins, Lipids, and Minerals. In J. Singh \& L. Kaur (Eds.), Advanced in Potato Biochemistry (pp. 99-125). Elsevier Academic Publishers, USA. http://dx.doi.org/10.1016/B978-0-12-374349-7.00005-2

Kiiya, W. W., Mureithi, J. G., \& Kiama, J. M. (2006). Improving production of irish potato (Solanum tuberosum L.) in Kenya: The use of green manure legumes for soil fertility Improvement. In J. G. Mureithi et al. (Eds.) Development and up scaling of green manure legumes technologiesin Kenya. KARI, Nairobi, Kenya.

Mehlich, A. (1953). Determination of $\mathrm{P}, \mathrm{Ca}, \mathrm{Mg}, \mathrm{K}, \mathrm{Na}$ and $\mathrm{NH}_{4}$ by the North Carolina Soil Testing Laboratory. Raleigh: North Carolina State University (Mimeograph).

Muthoni, J., \& Kabira, N. J. (2010). Effects of crop rotation soil macro content and pH in potato producing areas in Kenya. Case study of KARI Tigoni Station. Journal of Soil Science and Environmental Management, 1(9), 227-233.

Okalebo, J. R. (1985). A simple wet ashing technique of P, K, Ca and Mg analysis of plant tissue in a single digest. Kenya Journal of Science and Technology, B6, 129-133.

Recke, H., Schnier, H. F., Nabwile, S., \& Qureshi, J. N. (1997). Responses of Irish potatoes (Solanum tuberosum L.) to mineral and Organic fertilizer in various agro-ecological environments in Kenya. Exp. Agric., 33, 91-102. http://dx.doi.org/10.1017/S0014479797000203

Singer, J. M., \& Munns, N. D. (1987). Soils an Introduction. Macmillan Publishing Company, USA.

Tan, H. K. (2005). Soil Sampling, Preparation and Analysis (2nd ed.). CRC Press, Taylor \& Francis.

Walingo, L., Vark, W., Van Houba, V. J. G., \& Lee, J. J. V. (1989). Soil and plant analysis (Part 7). Plant analysis procedures. Syllabus. Wageningen Agricultural University, Neatherlands. 
White, P. J., \& Broadley, M. R. (2009). Bio fortification of crops with seven mineral elements often lacking in human diets - iron, zinc, copper, calcium, magnesium, selenium and iodine. New Phytologist, 182, 49-84. http://dx.doi.org/10.1111/j.1469-8137.2008.02738.x

WHO. (2004). Vitamin and Mineral Requirements in Human Nutrition (2nd ed.). World Health Organization and Food and Agriculture Organization of the United Nations.

Woolfe, A. J. (1987). The potato in the human diet. The Press Syndicate of the University of Cambridge. http://dx.doi.org/10.1017/CBO9780511753435

Abbreviations: Al, DLS, DAP, $\mathrm{Ca}, \mathrm{Cu}, \mathrm{Fe}, \mathrm{K}, \mathrm{Mg}, \mathrm{Mn}, \mathrm{Na}, \mathrm{P}, \mathrm{Zn}$

\section{Copyrights}

Copyright for this article is retained by the author(s), with first publication rights granted to the journal.

This is an open-access article distributed under the terms and conditions of the Creative Commons Attribution license (http://creativecommons.org/licenses/by/3.0/). 